\title{
Monitoring Insecticide Resistance and Target Site Mutations of L1014 Kdr And G119 Ace Alleles in Five Mosquito Populations in Korea
}

\author{
Seo Hye Park ${ }^{1, \dagger}$, Hojong Jun ${ }^{1, \dagger}$, Seong Kyu Ahn ${ }^{1}$, Jinyoung Lee ${ }^{1}$, Sung-Lim Yu², Sung Keun Lee ${ }^{3}$, Jung-Mi Kang, \\ Hyunwoo Kim ${ }^{6}$, Hee-ll Lee ${ }^{6}$, Sung-Jong Hong ${ }^{7} \mathbb{D}$, Byoung-Kuk Na ${ }^{4,5, *}$, Young Yil Bahk ${ }^{8, *}$, Tong-Soo Kim ${ }^{1, *}$ \\ ${ }^{1}$ Department of Parasitology and Tropical Medicine \& Global Resource Bank of Parasitic Protozoa Pathogens, Inha University School of Medicine, \\ Incheon 22212, Korea; ${ }^{2}$ Inha Research Institute for Medical Sciences, Inha University School of Medicine, Incheon 22212, Korea; ${ }^{3}$ Department of \\ Pharmacology, Inha University School of Medicine, Incheon 22212, Korea; ${ }^{4}$ Department of Parasitology and Tropical Medicine, and Institute of \\ Health Sciences, Gyeongsang National University College of Medicine, Jinju 52727, Korea; ${ }^{5}$ Department of Convergence Medical Science, \\ Gyeongsang National University, Jinju 52727, Korea; 'Division of Vectors and Parasitic Diseases, Korea Centers for Disease Control and Prevention, \\ Osong 28159, Korea; ' Department of Medical Environmental Biology, Chung-Ang University College of Medicine, Seoul 06974, Korea; ${ }^{8}$ Department \\ of Biotechnology, College of Biomedical and Health Science, Konkuk University, Chungju 27478, Korea
}

\begin{abstract}
Mosquitoes are globally distributed and important vectors for the transmission of many human diseases. Mosquito control is a difficult task and the cost of preventing mosquito-borne diseases is much lower than that for curing the associated diseases. Thus, chemical control remains the most effective tool for mosquito. Due to the long-term intensive use of insecticides to control mosquito vectors, resistance to most chemical insecticides has been reported. This study aimed to investigate the relationship between insecticide resistance and target site mutation of L1014 kdr and G119 ace alleles in 5 species/species group of mosquitoes (Aedes vexans, Ae. albopictus, Anopheles spp., Culex pipiens complex, and $C x$. tritaeniorhynchus) obtained from 6 collection sites. For Anopheles spp., the proportion of mosquitoes with mutated alleles in L1014 was 88.4\%, homozygous resistant genotypes were observed in $46.7 \%$, and heterozygous resistant genotypes were observed in $41.8 \%$. For the $C x$. pipiens complex and $C x$. tritaeniorhynchus species, homozygous resistant genotypes were found in $25.9 \%$ and $9.8 \%$, respectively. However, target site mutation of L1014 in the Ae. vexans nipponii and Ae. albopictus species was not observed. Anopheles spp., Cx. pipiens complex, and Cx. tritaeniorhynchus mosquitoes were resistant to deltamethrin and chlorpyriphos, whereas Ae. vexans nipponii and Ae. albopictus were clearly susceptible. We also found a correlation between the resistance phenotype and the presence of the L1014 kdr and G119 ace mutations only in the Anopheles spp. population. In this study, we suggest that insecticide resistance poses a growing threat and resistance management must be integrated into all mosquito control programs.
\end{abstract}

Key words: $k d r$, ace, insecticide resistance, mutation

\section{INTRODUCTION}

Global warming affects the survival and density of poikilothermic animals, including insects. The temperature in Korea is rising rapidly due to climate change [1]. Climate change is a major factor in mosquito ecology and provides conditions that are more favorable for the prevalence of mosquito-borne

- Received 30 May 2020, revised 31 August 2020, accepted 1 September 2020.

*Corresponding authors (bkna@gnu.ac.kr; bahk12@empal.com; byoung1@kku.ac.kr; tongsookim@inha.ac.kr)

†These authors contributed equally to this work.

(c) 2020, Korean Society for Parasitology and Tropical Medicine

This is an Open Access article distributed under the terms of the Creative Commons Attribution Non-Commercial License (https://creativecommons.org/licenses/by-nc/4.0) which permits unrestricted non-commercial use, distribution, and reproduction in any medium, provided the original work is properly cited. diseases along with new urban development or population growth [2]. In addition, countermeasures for infectious diseases must be prepared to prevent foreign mosquito-borne diseases from entering Korea [2-4].

In this sense, vector control of mosquitoes has become a pivotal portion of the global strategy to manage mosquito-associated diseases, especially malaria, Japanese encephalitis, West Nile encephalitis, and dengue fever. In this effort, insecticides, such as chlorpyriphos $(\mathrm{O}, \mathrm{O}$-diethyl $\mathrm{O}$-(3,5,6-trichloropyridin-2-yl) phosphorothioate) and pyrethroids, are the most important tool. Chlorpyriphos is a broad-spectrum organophosphate insecticide widely used as a pesticide to control a variety of insects [4]. Pyrethroids, which are analogous to naturally occurring pyrethrins, are a class of insecticides com- 
monly used for indoor spraying against mosquitoes due to their insecticidal potency and environmental stability as well as safety $[5,6]$. Although massive usage of insecticides was highly effective in controlling insect-borne diseases in the past decades, the widespread development of resistance in insects, especially mosquitoes, is now causing serious issues in many regions [7-9].

After prolonged exposure to an insecticide over several generations, mosquito populations may evolve resistance against the insecticide. Since mosquitoes can produce many generations per year, high levels of resistance can evolve very quickly. It has been shown that multiple resistance mechanisms are specific to individual mosquito species $[10,11]$. Overall, insecticide resistance is not only conferred via multiple mechanisms as well as reduced cuticle penetrance, but also mediated through the interaction of regulatory genes and resistance genes. However, it is not evident which and how various genes are involved.

The knockdown resistance $k d r$ gene is the primary mechanism responsible for resistance to dichlorodiphenyltrichloroethane (DDT) and pyrethroids [12-14]. The enzyme synaptic acetylcholinesterase (AChE1) is the target of organophosphorus and carbamate, which are competitive inhibitors of acetylcholine $[15,16]$. Genomic studies commonly focus on single nucleotide polymorphisms because they can be readily identified with short region sequencing. However, it is evident that structural variants, including inversions, duplications, and deletions may be involved in adaptation and speciation within mosquitoes $[17,18]$. In this study, we examined sequence variations in specific genes, including knockdown resistance $(k d r)$ (L1014 in domain II segment 6) and acetylcholine esterase (ace) (G119 region) collected from mosquitoes at various sampling locations comprising Ae. vexans nipponii, Ae. albopictus, Anopheles spp., Cx. pipiens complex, and Cx. tritaeniorhynchus.

\section{MATERIALS AND METHODS}

\section{Mosquito collection}

In general, mosquitoes have different insecticide resistances depending on their habitat. Thus, mosquito collection sites for this study were considered depending on the type of mosquito habitat. Adult mosquitoes were collected using unbaited blacklight (BL) traps (SC-2000, Shinyeong Co., Namyangju, Korea), BG-Sentinel ${ }^{\mathrm{TM}}$ (BGS) traps (Biogents, Regenburg, Germany), or hand-held battery-powered aspirators at each sample collec- tion site in summer 2017 and 2018. The mosquitoes were identified and classified according to morphology-based specialized taxonomic keys [19-22] and compared with standard specimens in the collection of the School of Medicine, Inha University, and the Korea Centers for Diseases Control and Prevention (KCDC). Collections were made over a minimum of 2 days at each site. The collected mosquitoes were pooled by species/species group, collection site, and time of survey, and quickly transferred to microcentrifuge tubes at $4^{\circ} \mathrm{C}$ where the species/species group were identified. The mosquitoes were assayed to examine target site sequence variations in specific genes, including knockdown resistance ( $k d r$, L1014 in domain II) and acetylcholine esterase (ace, G119). For insecticide resistance bottle assays, living mosquitoes were transferred to the School of Medicine, Inha University, as quickly as possible and assayed according to the standard method described below in the section "insecticide resistance bottle assays". Table 1 lists the geographic locations, collection methods, and numbers of the collected species/species group of mosquitoes. A total of 7,720 adult mosquitoes comprising the 5 species/species group of interest (Ae. vexans nipponii $(\mathrm{n}=1,708)$, Ae. albopictus ( $\mathrm{n}=955)$, Anopheles spp. $(\mathrm{n}=2,744)$, Cx. pipiens complex $(\mathrm{n}=1,685)$, and $C x$. tritaeniorhynchus $(\mathrm{n}=628)$, which were identified and classified according to morphology-based specialized taxonomic keys $[19,20]$, were collected in summer 2017 and 2018 and the target site mutations were evaluated. L1014F $k d r$ and G119S ace mutations were analyzed in 1,010 and 1,258 mosquitoes, respectively.

\section{Insecticide resistance bottle bioassays}

Adult mosquitoes were collected, were kept in cooled cages to increase survival and provided with $10 \%$ sucrose solution ad libitum until the time of testing. The bottle bioassays followed the KCDC and World Health Organization (WHO) guidelines [23]. In brief, mosquitoes were placed in a $250 \mathrm{ml}$ glass Wheaton bottle coated with $1.25 \mathrm{mg} / \mathrm{ml}$ of deltamethrin (Pyrethroid) and chlorpyrifos (Organophosphate) diluted in acetone. The negative control bottle was treated with acetone only. Bottles were swirled so that the glass bottom and inside cap were coated and rotated while rocking so that the side were evenly coated with insecticide. An aspirator was used to gently add 25 mosquitoes into each bottle. Afterward, knockdown mosquitoes were recorded every $30 \mathrm{~min}$ for $6 \mathrm{hr}$. The exposure time for diagnosis resistance of each insecticide was set to $30 \mathrm{~min}$. Collected mosquitoes were transferred into 


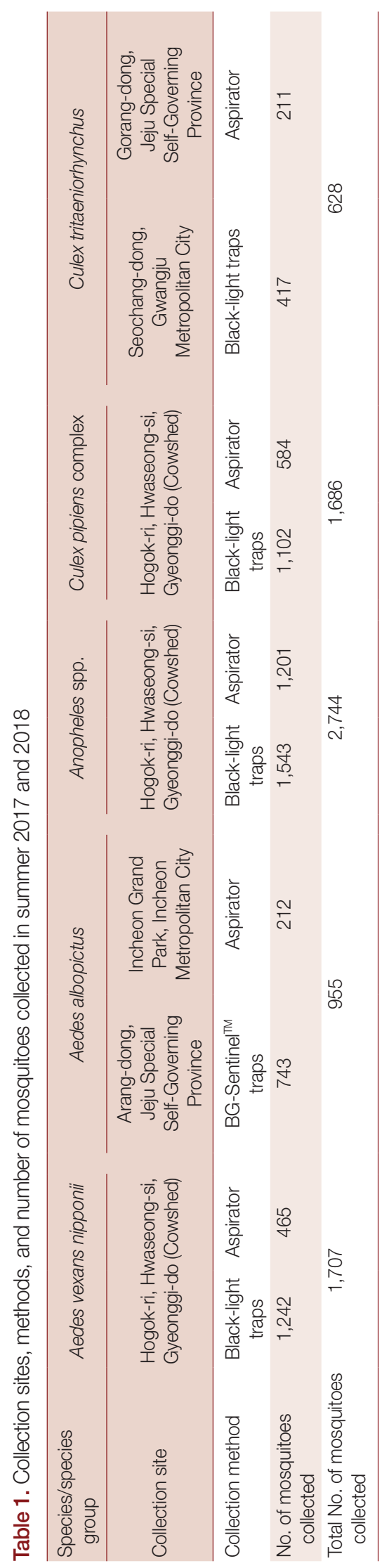

Wheaton bottle coated with each insecticide and incubate for $30 \mathrm{~min}$. After the incubation periods, all living mosquitoes were placed in a pint-sized paper cage with constant access to a $10 \%$ sucrose solution. The mortality was assessed after $24 \mathrm{hr}$. Surviving individuals were frozen at $-70^{\circ} \mathrm{C}$ for molecular analysis.

\section{Isolation of genomic DNA from mosquitoes.}

Mosquito genomic DNA was extracted individually using the QIAamp DNA mini kit (Qiagen GmbH, Hilden, Germany) according to the manufacturer's protocol. Briefly, the entire body of a single mosquito was homogenized in a microcentrifuge tube containing $80 \mu \mathrm{l}$ of PBS using a pestle for $30 \mathrm{sec}$. The homogenized samples were incubated with $20 \mu \mathrm{l}$ of proteinase $\mathrm{K}$ and $100 \mu \mathrm{l}$ of ATL buffer at $56^{\circ} \mathrm{C}$ for $2 \mathrm{hr}$ until completely lysed with occasional vortexing during incubation. After centrifuging quickly, $200 \mu \mathrm{l}$ of AL buffer was added to the mixture followed by incubation at $70^{\circ} \mathrm{C}$ for $10 \mathrm{~min}$ and $200 \mu \mathrm{l}$ of absolute ethanol was added with vigorous vortexing for thorough mixing. After brief centrifugation, the mixtures were applied to the QIAamp Mini Spin column. The mixture-applied column was washed with $1 \mathrm{ml}$ of AW1 buffer followed by centrifugation at $6,000 \times \mathrm{g}$ for $1 \mathrm{~min}$ and then with $1 \mathrm{ml}$ of AW2 buffer followed by centrifuging at maximum speed for $3 \mathrm{~min}$. The column-bound DNA was eluted with $150 \mu \mathrm{l}$ of distilled water by centrifugation at $6,000 \times \mathrm{g}$ for $1 \mathrm{~min}$. The concentration of the extracted DNA was determined by measuring ultraviolet absorbance at $260 \mathrm{~nm}$ using a spectrophotometer (NanoDrop $^{\mathrm{TM}}$ ND-1000, Thermo Fisher Scientific, Lafayette, Colorado, USA), after which the samples were stored at $-70^{\circ} \mathrm{C}$ before use.

\section{Detection of L1014 and G119 mutations}

The primer set sequences and reaction conditions for the amplification of $k d r$ and ace genes for each targeted mosquito are listed in (Supplementary Table S1). The specificity of the primers was confirmed using a BLAST search in the GenBank database from NCBI. PCR parameters were as follows: a denaturation step at $94^{\circ} \mathrm{C}$ for $5 \mathrm{~min}$ and then 30 cycles of a denaturation step at $94^{\circ} \mathrm{C}$ for $1 \mathrm{~min}$, an annealing step at $52^{\circ} \mathrm{C}$ for 1 min, and extension at $72^{\circ} \mathrm{C}$ for $1 \mathrm{~min}$, with a final extension at $72^{\circ} \mathrm{C}$ for $10 \mathrm{~min}$. The PCR products were analyzed by electrophoresis in $1.5 \%$ agarose gel. The amplified DNA fragments were cloned into TA cloning vector (Promega Corporation, Madison, Wisconsin, USA) and sequenced by Macrogen Inc. 
(Seoul, Korea). The DNA sequences were analyzed using DNASTAR and the NCBI-BLAST service.

\section{RESULTS}

\section{Genotyping of target site mutations: $k d r$ and ace genes}

In this study, we investigated the status of resistance in 5 common mosquitoes (Ae. vexans nipponii, Ae. albopictus, Anopheles spp., $C x$. pipiens complex, and $C x$. tritaeniorhynchus) collected from different locations in Korea (Table 1). L1014F $k d r$ and G119S ace mutations were analyzed in 1,010 and 1,258 mosquitoes, respectively. The frequency of target site mutations is presented in Table 2. kdr target site mutations in 1,010 mosquito samples were analyzed by sequencing of the Domain II region. For the L1014F $k d r$ target site mutations, homozygous resistant genotypes (L1014F/L1014F and L1014C/L1014C) were observed in $17.5 \%(177 / 1,010)$ and in $0.7 \%(7 / 1,010)$ of 1,010 tested samples, respectively. Homozygous resistant genotypes (L1014C/L1014F and L1014/L1014F) were found in 5.5\% $(56 / 1,010)$ and $5.2 \%(53 / 1,010)$, respectively. A homozygous susceptible genotype (L1014/L1014) was found in 195 $(19.3 \%)$ mosquitoes from a total of 1,010 sequenced samples. For Anopheles spp., the rate of mosquitoes with mutated alleles in the L1014 $k d r$ gene was $88.4 \%$ (199/225 sequenced samples of Anopheles spp.), homozygous resistant genotypes were observed in 46.7\% (105/225), and heterozygous resistant genotypes (L1014/L1014F and L1014C/L1014F) were observed in $41.8 \%(94 / 225)$. In practice, since it is difficult to classify species group of the genus Anopheles by morphological differences alone and it is hard to perform the PCR experiments for each Anopheles subspecies mosquito, we categorized mosquitoes in this genus as simply just Anopheles spp. It has been reported that there are various Anopheles subspecies in Korea including Anopheles lesteri, An. sinensis, An. pullus, An. kleini, and An. belenrae [24].

For $C x$. pipiens complex and $C x$. tritaeniorhynchus, homozygous resistant genotypes were found in $25.9 \%$ (68/263) and in $9.8 \%$ (11/112) of samples, respectively. Heterozygous resistant genotypes were found in 13.4\% (15/112) of Cx. tritaeniorhynchus samples (Table 2). In addition, various point mutations in the Domain II region were amplified and analyzed in this study, including M976V, L982P, C983R, W986R, N1013D, and L1025M. However, as expected, target site mutation for L1014 in the kdr Domain II region was not observed in the Ae. vexans nipponii and Ae. albopictus species.

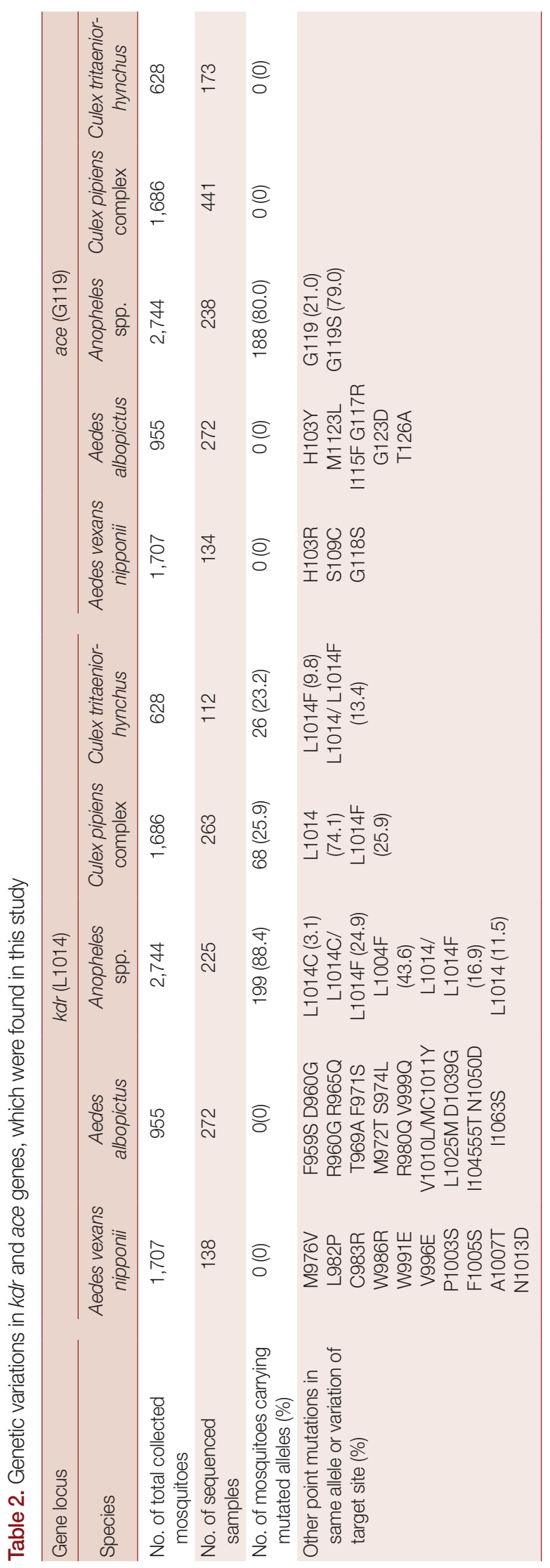


For the G119 ace target site mutation, the individuals with the homozygous susceptible genotype (G119) comprised 21\% (50/238 sequenced samples in Anopheles spp.) and those with the homozygous resistant genotype (G119S) comprised 79\% (188/238) (Table 2). However, target site mutations of G119 in the ace gene in the Ae. vexans nipponii, Ae. albopictus, Cx. pipiens complex, and $C x$. tritaeniorhynchus species were not observed in this study. In addition, various point mutations in the ace gene were amplified and analyzed, including H103R, S109C, and G118S, especially in the Culex genus.

\section{Insecticide susceptibility bioassays}

Insecticide resistance bottle bioassays showed that the mosquitoes collected from the study areas exhibited 0-61.0\% resistance to deltamethrin and chlorpyriphos. Ae. vexans nipponii and Ae. albopictus from the study areas showed no resistance to the insecticides tested (pyrethroids (deltamethrin) and organophosphates (chlorpyriphos)), which were used as target insecticides for L1014 $k d r$ and G119 ace genes, respectively (Table 3). However, resistance was observed in a mosquito species group, Anopheles spp., with mortality rates of 86.1\% (537/624 tested mosquitoes) for deltamethrin and $66.6 \%$ (384/577) for chlorpyriphos. Deltamethrin resistance was also observed in the mosquito species $C x$. pipiens complex and $C x$. tritaeniorhynchus, with mortality rates of $78.3 \%$ (267/341) and 53.8\% (50/93), respectively, but resistance to chlorpyriphos was not observed at all. After insecticide bioassays, the insecticide-resis- tant mosquitoes exposed to deltamethrin or chlorpyriphos were tested using PCR to identify the target site mutations for L1014 $k d r$ and G119 ace genes (Table 3). The mosquitoes used for the bioassays with deltamethrin (pyrethroids) (204 specimens) and chlorpyriphos (organophosphate) (316 specimens) were evaluated. The species/species group with the highest frequency of insecticide-resistance was Anopheles spp., with 78\% (68/87 resistant mosquitoes) resistance to deltamethrin, followed by the same species group with 69\% (133/193) resistance to chlorpyriphos, and $C x$. pipiens complex and $C x$. tritaeniorhynchus with $14 \%$ (10/74, and 6/43, respectively) resistance to deltamethrin. In this sense, the diagnostic test using $k d r$ and ace as resistance markers in 5 species/species groups of mosquitoes revealed differences between deltamethrin and chlorpyriphos, with a high specificity for both (78\% and 69\%, respectively) especially in Anopheles spp. Cx. pipiens complex and $C x$. tritaeniorhynchus populations resistant to chlorpyriphos were not observed. These results indicate that the presence of the L1014 $k d r$ and G119 ace gene mutations was significantly associated with resistance to the 2 tested insecticides in Anopheles spp. However, with the exception of Anopheles spp. resistance to the 2 insecticides tested, no significant correlation was observed between target site mutations in the L1014 kdr and G119 ace genes and the resistance phenotype in the tested mosquito species/species group.

Table 3. Mutation frequency of $k d r$ and ace genes in the mosquito (resistant/susceptible) phenotype grouped using insecticide resistance bioassay

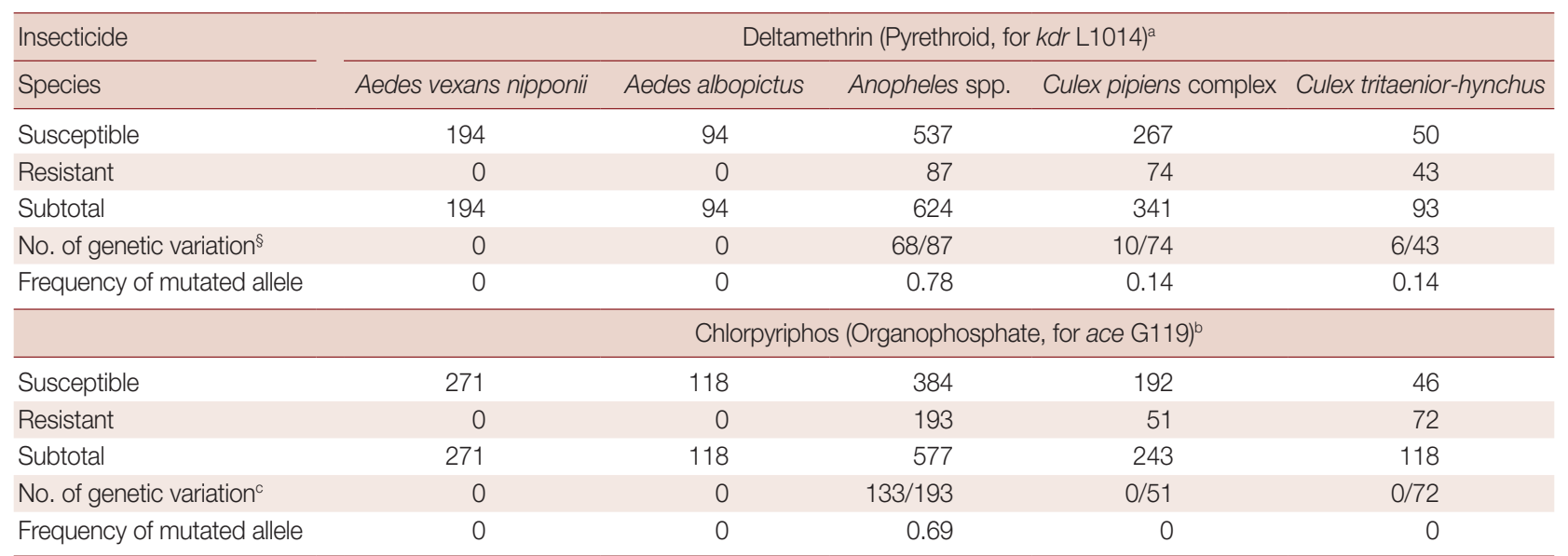

Test for insecticide resistance with L1014 kdr gene.

'Test for insecticide resistance with G119 ace gene.

'No. of mosquitoes with mutated alleles in L1014 or G119/No. of surviving mosquitoes in bioassays. Because the control mortality is <5\%, this study was not corrected using Abbot's formula. 


\section{DISCUSSION}

Mosquitoes are important vectors of diseases. The absence of effective vaccines forces the use of insecticides as the major tool to control vector populations $[25,26]$. Mosquitoes were collected from 6 locations nationwide to ensure local diversity of the mosquito species/species group, and the habitat characteristics for each mosquito species/species group were taken into consideration. In general, Ae. albopictus is considered a forest mosquito, and therefore has had minimal exposure to insecticides sprayed countless times in rice paddies and fields, resulting in low resistance against insecticides. However, Anopheles spp. mosquitoes, which are found in rice paddies and are therefore exposed to agricultural pesticides sprayed each year, are thought to be more resistant than Ae. albopictus. Insecticide resistance bottle bioassays showed that the mosquitoes collected from the study areas exhibited resistance to deltamethrin and chlorpyriphos. Especially, Ae. vexans nipponii and Ae. albopictus species showed $100 \%$ susceptibility to the 2 tested insecticides. However, $C x$. tritaeniorhynchus mosquitoes showed the highest resistance level of $46.2 \%$ to deltamethrin and $61.0 \%$ to chlorpyriphos. Anopheles spp. exhibited a resistance level of $13.9 \%$ to deltamethrin and $33.4 \%$ to chlorpyriphos while the $C x$. pipiens complex exhibited $21.7 \%$ resistance to deltamethrin and $21.0 \%$ to chlorpyriphos (Table 3).

Grasping the mechanisms underlying these insecticide resistances is critical to understanding the optimal method to apply these chemicals and conserve their efficacy as tools to control vector populations. In addition, we also examined the well-known and widely tested insecticide target site mutations in L1014 of domain II segment 6 in the kdr and G119 ace genes. The L1014F substitution mutation confers target site insensitivity and has been reported in $C x$. pipiens mosquitoes [27]. Other mutations including L1014S and L1014C were also reported in various regions [28-31]. Both L1014F $k d r$ and L1014H/C/S/W kdr-type resistances have been identified in numerous species [32]. However, it has been reported that many mosquitoes that present a resistant phenotype also carry the L1014F $k d r$ allele and the $k d r$ site mutation may not be a unique mechanism that confers resistance to pyrethroids and DDT [33]. G119S ace gene mutation is responsible for the reduction of AChE1 activity in cholinergic synapses and is one of the most common mutations detected in Cx. pipiens mosquitoes [34]. An obvious correlation between the degrees of resistance and the frequency of insecticide usage has been well-established in the Cx. pipiens population [35]. Our findings indicated that the G119S ace allele was present only in the tested Anopheles spp. population, which presented a resistant phenotype to chlorpyriphos. Chlorpyriphos, as well as temephos and pyrimiphos-methyl, are widely used in the control of mosquito larvae [36].

There are 2 ways to control mosquito-borne diseases; the first is by administering medicine to infected individuals and the second is by controlling vector mosquitoes. Chemical control for mosquito elimination is still the most efficient primary tool. However, due to the large scale and intensive application of insecticides to control mosquito vectors over many years, resistance to most chemical insecticides has been reported. After the US military was stationed in the Southern part of the Korean Peninsula in 1940's, DDT solution was sprayed in the region to prevent the outbreak of diseases carried by flies and mosquitoes in US military stations [37]. After this period, extensive use of agricultural pesticides also contributed greatly to the decrease in disease incidence through the control of the mosquito vector of the disease. Due to the nature of their habitat environment, Ae. vexans nipponii and Ae. albopictus are thought to have a lower probability of exposure to insecticides than other species. The habitats of these species are usually forests, parks, or quarantine regions, and they generally live out of reach of people and are therefore more susceptible to insecticides. In contrast, other species of mosquitoes examined in this study are continuously exposed to various insecticides. This continuous and intensive exposure to insecticides is responsible for the high levels of resistance. The resistance levels are not only due to intensive prior use but also due to the acquisition of cross-resistance [36]. Sinegre et al. [35] reported that in Cx. pipiens treated with chlorpyriphos, prolonged treatment led to the appearance of resistance to other organophosphates. In addition, it was confirmed that prolonged exposure to organophosphates always leads to the appearance of crossresistance to other organophosphates and sometimes to certain carbamate-family insecticides [38]. In addition, it is suggested that the high rates of resistance could be explained by the appearance of cross-resistance, especially when the area was treated only with temephos for larvae and pyrethroid for adults [36,39].

Our study demonstrated some correlations between the resistance phenotype and the presence of the L1014 kdr and G119 ace mutations in the Anopheles spp. population. However, there was no clear correlation between the insecticide resistance 
phenotype and target site mutation in the other 4 mosquito species tested. Target site mutation coupled with enzyme detoxification has been reported in An. gambiae and $C x$. quinquefasciatus from Benin [40]. Moreover, the involvement of metabolic resistance such as carboxylesterase, cytochrome P450 monooxygenase, and glutathione $S$-transferase requires further investigation to elucidate the mechanisms of resistance to the insecticides currently used in mosquito-vector control.

Insecticide-resistance bottle assays and molecular identification of target-site mutation of L1014 $k d r$ and G119 ace alleles clearly indicated that Anopheles spp., Cx. pipiens complex, and $C x$. tritaeniorhynchus mosquitoes were resistant to 2 tested insecticides, deltamethrin and chlorpyriphos, but Ae. vexans nipponii and Ae. albopictus were clearly susceptible. The frequencies of resistant $k d r$ and ace alleles containing L1014F or L1014C and G119S substitution mutations are high for Anopheles spp. populations collected at the monitoring sites. A correlation between the resistance phenotype and the presence of the L1014 $k d r$ and G119 ace mutations was found only in the Anopheles spp. population. Thus, it is clear that insecticide resistance poses a growing threat and resistance management must be integrated into all mosquito control programs.

\section{ACKNOWLEDGMENT}

This work was supported by the Inha University Research Fund and the Korea Centers for Disease Control and Prevention (2017NER44030) in the Republic of Korea.

\section{CONFLICT OF INTEREST}

The authors declare that they have no conflicts of interest.

\section{REFERENCES}

1. Korea Meteorological Administration (KMA). 2019 Abnormal Weather Report [Internet]; [cited 2020 Feb 20]. Available from: http://www.climate.go.kr/home/bbs/view.php?code=93\&bnam e $=$ abnormal $\&$ vcode $=6385 \&$ cpage $=1 \&$ vNum $=$ Notice $\&$ skind $=\&$ s word=\&category $1=\&$ category 2 .

2. Yeom JS. Current status and outlook of mosquito-borne diseases in Korea. J Korean Med Assoc 2017; 60: 468-474 (Korean).

3. Bahk YY, Lee HW, Na BK, Kim J, Jin K, Hong YS, Kim TS. Epidemiological characteristics of re-emerging vivax malaria in the Republic of Korea (1993-2017). Korean J Parasitol 2018; 56: 531543.

4. Korea Centers for Disease Control and Prevention. Epidemiology and Management of Vaccine Preventable Diseases. 5th eds. Cheongju, Korea. Korea Centers for Disease Control and Prevention. 2017.

5. Kharkongor M, Hooroo RNK, Dey S. Effects of the insecticide chlorpyrifos, on hatching, mortality and morphology of Duttaphrynus melanostictus embryos. Chemosphere 2018; 210: 917921.

6. Lu Z, Gan J, Cui X, Delgado-Moreno L, Lin K. Understanding the bioavailability of pyrethroids in the aquatic environment using chemical approaches. Environ Int 2019; 129: 194-207.

7. Hemingway J, Field L, Vontas J. An overview of insecticide resistance. Science 2002; 298: 96-97.

8. Ketterman AJ, Saisawang C, Wongsantichon J. Insect glutathione transferases. Drug Metab Rev 2011; 43: 253-265.

9. Zaim M, Guillet P. Alternative insecticides: an urgent need. Trends Parasitol 2002; 18: 161-163.

10. Liu N. Insecticide resistance in mosquitoes: impact, mechanisms, and research directions. Ann Rev Entomol 2015; 60: 537559.

11. Main BJ, Everitt A, Cornel AJ, Hormozdiari F, Lanzaro GC. Genetic variation associated with increased insecticide resistance in the malaria mosquito, Anopheles coluzzii. Parasit Vectors 2018; 11: 225.

12. Dong K. A single amino acid change in the para sodium channel protein is associated with knockdown-resistance $(k d r)$ to pyrethroids insecticides in German cockroach. Insect Biochem Mol Biol 1997; 27: 93-100.

13. Busvine JR. Mechanism of resistance to insecticide in houseflies. Nature 1951; 168: 193-195.

14. Soderlund DM, Knipple DC. The molecular biology of knockdown resistance to pyrethroids insecticides. Insec Biochem Mol Biol 2003; 33: 563-577.

15. Weill M, Lutfalla G, Mogensen K, Chandre F, Berthomieu A, Berticat C, Pasteur N, Philips A, Fort P, Raymond M. Comparative genomics: Insecticide resistance in mosquito vectors. Nature 2003; 423: 136-137.

16. Weill M, Malcolm C, Chandre F, Mogensen K, Berthomieu A, Marquine $\mathrm{M}$, Raymond $\mathrm{M}$. The unique mutation in ace-1 giving high insecticide resistance is easily detectable in mosquito vectors. Insect Mol Biol 2004; 13: 1-7.

17. Lanzaro GC, Touré YT, Carnahan J, Zheng L, Dolo G, Traoré S, Petrarca V, Vernick KD, Taylor CE. Complexities in the genetic structure of Anopheles gambiae populations in west Africa as revealed by microsatellite DNA analysis. Proc Natl Acad Sci USA 1998; 95: 14260-14265.

18. Schmidt JM, Good RT, Appleton B, Sherrard J, Raymant GC, Bogwitz MR, Martin J, Daborn PJ, Goddard ME, Batterham P, Robin C. Copy number variation and transposable elements feature in recent, ongoing adaptation at the Cyp6g1 locus. PLoS Genet 2010; 6: e1000998.

19. Tanaka K, Mizusawa K, Saugsad ES. A Revision of the Adult and Larval Mosquitoes of Japan (including the Ryukyu Archipelago and the Ogasawara Islands) and Korea (Diptera: Culicidae). Ann 
Arbor, USA. American Entomological Institute. 1979.

20. Lee KW. A Revision of the Illustrated Taxonomic Keys to Genera and Species of Female Mosquitoes of Korea. Seoul, Korea: Department of the Army, 5th Medical Detachment, 168th Medical Battalion, 18th Medical Command. 1998, pp 40.

21. Jegal S, Jun H, Kim-Jeon MD, Park SH, Ahn SK, Lee J, Gong YW, Joo K, Kwon MJ, Roh JY, Lee WG, Lee W, Bahk YY, Kim TS. Three-year surveillance of culicine mosquiotes (Diptera: Culicidae) for flavivirus infections in Incheon metropolitan city and Hwaseong-si of Gyeonggi-do province, Republic of Korea. Acta Trop 2020; 202: 105258.

22. Bahk YY, Jun H, Park SH, Jung H, Jegal S, Kim-Jeon MD, Roh JY, Lee WG, Ahn SK, Lee J, Joo K, Gong YW, Kwon MJ, Kim TS. Flaviviral disease mosquito vector surveillance in Incheon Metropolitan City and the Hwaseong area, Gyeonggi-do, Republic of Korea. Entomol Res 2020; 50: 3-13.

23. World Health Organization. Test Procedures for Insecticide Resistance Monitoring in Malaria Vector Mosquitoes [Internet]; [cited 2020 Mar 4] Available from: http://www.who.int/malaria/publications/ atoz/9789241511575/en/.

24. Foley DH, Klein TA, Lee IY, Kim MS, Wilkerson RC, Harrison G, Rueeda LM, Kim HC. Mosquito species composition and Plasmodium vivax infection rates on Baengyeong-do (Island), Republic of Korea. Korean J Parasitol 2011; 49: 313-316.

25. Zaim M, Aitio A, Nakashima N. Safety of pyrethroid-treated mosquito nets. Med Vet Entomol 2000; 14: 1-5.

26. World Wildlife Fund. Hazards and Exposures Associated with DDT and Synthetic Pyrethroids used for Vector Control. Toronto, Canada. World Wildlife Fund; 1999.

27. Martinez-Torres D, Chevillon C, Brun-Barale A, Bergé JB, Pasteur $\mathrm{N}$, Pauron D. Voltage-dependent $\mathrm{Na}^{+}$channels in pyrethroidresistant Culex pipiens L. mosquitoes. Pest Manag Sci 1999; 55: 1012-1020.

28. Xu Q, Tain L, Zhang L, Liu N. Sodium channel genes and their differential genotypes at the F-to-F $k d r$ locus in the mosquito Culex quinquefasciatus. Biochem Biophys Res Comm2011; 40: 645-649.

29. Wang ZM, Li CX, Xing D, Yu YH, Liu N, Xue RD, Dong YD, Zhao TY. Detection and widespread distribution of sodium channel alleles characteristic of insecticide resistance in Culex pipiens complex mosquitoes in China. Med Vet Entomol 2012; 26: 228-232.

30. Diabate A, Brengues C, Baldet T, Dabiré KR, Hougard JM, Akogbeto M, Kengne P, Simard F, Guillet P, Hemingway J, Chandre F. The spread of the Leu-Phe kdr mutation through Anopheles gambiae complex in Burkina Faso: genetic introgression and de novo phenomena. Tropical Med Int Health 2004; 9: 1267-1273.

31. Scott JG, Yoshimizu M, Kasal S. Pyrethroid resistance in Culex pipiens mosquitoes. Pest Biochem Physiol 2015; 120: 68-76.

32. Brooke BD. $k d r$ : can a single mutation produce an entire insecticide resistance phenotype? Trans R Soc Trop Med Hyg 2008; 102: 524-525.

33. Rinkevich FD, Du Y, Dong K. Diversity and convergence of sodium channel mutations involved in resistance to pyrethroids. Pestic Biochem Physiol 2013; 106: 93-100.

34. Weill M, Lutfalla G, Mogensen K, Chandre F, Berthomieu A, Berticat C, Pasteur N, Philips A, Fort P, Raymond M. Comparative genomics: insecticide resistance in mosquito vectors. Nature 2003; 423: 136-137.

35. Sinegre G, Jullian J, Gaven B. Acquisition progressive de la résistance a chlorpyiphos chez les larves de Culex pipiens (L) dans le midi la France. Parasitologia 1977; 19: 1-2.

36. Tmimi FZ, Faraj C, BkhacheM, Mounaji K, Failloux AB, Sarih M. Insecticide resistance and target site mutations (G119S ace-1 and L1014F kdr) of Culex pipiens in Morocco. Parasit Vectors 2018; 11: 51.

37. Chavasse DC, Yop HH. Chemical Methods for the Control of Vectors and Pests of Public Health Importance, Vol. 2. Geneva, Switzerland. World Health Organization. 1997, pp 129.

38. Yeo IS. U.S. military administration's malaria control activities (1945-1948). Korean J Med Hist 2015; 24: 35-65.

39. Yadouléton A, Badirou K, Agbanrin R, Jöst H, Attolou R, Srinivasan R, Padonou G, Akogbéto M. Insecticide resistance status in Culex quinquefasciatus in Benin. Parasit Vectors 2015; 8: 17.

40. Antonio-Nkondjio C, Poupardin R, Tene BF, Kopya E, Costantini $\mathrm{C}$, Awono-Ambene P, Wondji CS. Investigation of bendiocarb resistance in Anopheles gambiae populations from the city of Yaoundé, Cameroon. Malar J 2016; 15: 424. 